\title{
Bioactive Peptides
}

\section{(J. Howl and S. Jones, eds., CRC Press, Taylor \& Francis Group, Boca Raton-London-New York, 2009, 479 p., \$143.96)}

DOI: $10.1134 / \mathrm{S} 0006297910050214$

This book contains four parts and 19 chapters. Part I highlights receptor ligands and consists of 12 chapters. The 1st chapter is devoted to angiotensins: from endocrine to intracrine functions. There is consideration of the sources of angiotensin peptides, their molecular actions, intracrine actions, structural analogs, and common methodologies and assay systems. Separate parts of this chapter include measurement of angiotensin peptides and peptidase activities. The final part discusses clinical applications and future developments related to angiotensins.

Chapter 2 contains data about calcitonin (CT), amylin, calcitonin gene-related peptide, and adrenomedullins. Also, related peptides known as CT receptorstimulating peptides are characterized.

Chapter 3 highlights the neuropeptide $\mathrm{Y}$ family (NPYF) and their receptors. NPYF includes three different 36-amino-acid-long peptide members: neuropeptide $\mathrm{Y}$ (NPY), which is expressed in the central and peripheral nervous systems, peptide YY (PYY), and pancreatic peptide (PP). NPY and PYY have 70\% sequence similarity, whereas PP exhibits only 50\% homology with NPY. Although structure of these peptides is very similar, their functions are distinct.

Chapter 4 contains data about pituitary adenylate cyclase-activating polypeptide including biological and molecular mechanism of action, structural analogs, assay systems, and perspective and future development.

Chapter 5 describes opioid peptides from mammalian, amphibian, and other sources. There is discussion on molecular mechanism of action, structural analogs of opioid peptides, and their assay systems.

Chapter 6 focuses on cholecystokinins and gastrins. There is analysis of cellular synthesis of these peptides, their structural analogs, and common assay systems.
Chapter 7 is devoted to the chemistry and biology of insulin-like peptide 3, a novel member of the insulin superfamily.

In chapter 8 there is discussion on somatostatin analogs including their clinical applications and side effects.

Chapter 9 highlights tachykinins, the largest known peptide family responsible for a range of pleiotropic functions in both vertebrates and invertebrates. The tachykinins are almost exclusively peptides of neuronal origin, and they also were found in a range of peripheral cells and tissues, including immune and inflammatory cells and even in the placenta. They are involved in a wide range of biological processes such as immunomodulation, neurogenic inflammation and nociception, smooth muscle contraction, vasodilatation, hematopoiesis, and stimulation of endocrine gland secretion.

Chapters 10-12 highlight neurotensin, galanin, and bioactive peptides in gut-brain signaling.

Part II includes only one chapter, chapter 13, which focuses on nonribosomal peptides.

Part III (chapters 14-16) summarizes data about host-defense peptides and antimicrobials.

Part IV (chapters 17-19) highlights venom components and toxins. There are discussions on mast cell degranulating peptides, mastoparans, and spider venom and hemolymph-derived cytolytic and antimicrobial peptides.

Each chapter contains a basic bibliography related to book topics, and there is a subject index at the end of the book.

The book will be useful for researchers in protein chemistry and biochemistry, molecular biologists and physicians, and teachers and students of medical schools and universities specialized in proteomics. 